\title{
Esquemas terapêuticos encurtados para o tratamento de malánia por Plasmodium vivax
}

\author{
Short course schemes for vivax malaria treatment \\ Rita do Socorro Uchôa da Silva', Ana Yecê das Neves Pinto', \\ Vanja Suely Pachiano Calvosa ${ }^{1}$ e José Maria de Souza ${ }^{1}$
}

\begin{abstract}
Resumo Visando avaliar esquemas terapêuticos encurtados eficazes no tratamento de malária vivax, foi realizado um estudo aberto, prospectivo, alocando 234 pacientes com malária por P. vivax, distribuídos aleatoriamente em 8 grupos terapêuticos. Seis grupos usaram como esquizonticida sangüíneo o artesunato via oral em diferentes dosagens por um dia e aos outros dois grupos foi administrada a cloroquina em dose única. Como hipnozoiticida, foi utilizada a primaquina em dose diária de $30 \mathrm{mg}$ dia durante cinco ou sete dias, em ambos os grupos. O desaparecimento da parasitemia nos pacientes tratados com artesunato (independente da dose) foi mais rápido quando comparados aos que fizeram uso de cloroquina $(p<0,01)$. Cura ocorreu em $92,3 \%$ e $80,2 \%$, respectivamente nos pacientes tratados com primaquina por sete e cinco dias $(p=0,0372)$, independente do esquizonticida sanguíneo utilizado.
\end{abstract}

Palavras-chaves: P. vivax. Tratamento. Cloroquina. Artesunato. Primaquina.

Abstract with the objective of evaluating shortened therapeutic outlines effective in vivax malaria treatment, we accomplished an open, prospective study allocating 234 patients with vivax malaria distributed at random into eight therapeutic groups. Six groups used oral arthemisin as blood esquizonticide at different doses for one day and the other two groups received chloroquine in a single dose. The primaquine was used as a hypnozoiticide in all groups. They received a daily dose of $30 \mathrm{mg}$ in the course of five or seven days in all groups. The clearance of parasitaemia in patients treated with arthemisin (independent of dosage) was faster than the chloroquine group $(p<0.01)$. Cure was acheived in $92.3 \%$ and $80.2 \%$, in patients treated with primaquine for seven or five days, respectively $(p=0.0372)$.

Key-words: Vivax malaria. Treatment. Chloroquine. Arthemisin. Primaquine.

O controle de malária no Brasil tem encontrado inúmeros obstáculos, entre eles, a prolongada duração do tratamento, principalmente em malária por $P$. vivax, cujas formas latentes do ciclo hepático (hipnozoítos) podem persistir viáveis mais freqüentemente em tratamentos de duração menor que cinco dias, quando comparados aos esquemas de maior duração ${ }^{11315}$.

Logo após as primeiras doses de antimaláricos, os portadores de malária vivax, tornam-se assintomáticos e tendem a abandonar o tratamento, usando a primaquina por um tempo menor. A primaquina é droga essencial para a determinação da cura radical e evita uma série de problemas aos Programas de Controle de Malária, tais como: gastos com novo tratamento e permanência de fontes de infecção anofélica (homem doente).

Os derivados da artemisinina constituem excelentes esquizonticidas sangüíneos, tornando o paciente assintomático rapidamente, determinando raros ou nenhum efeito colateral quando administrados aos pacientes com malária pelo $P$. falciparum.

Mosquitos da espécie Anopheles darlingi, principal vetor da doença no Brasil, podem durante o repasto

1. Instituto Evandro Chagas da Fundação Nacional de Saúde do Ministério da Saúde, Belém, PA.

Órgãos financiadores: Instituto Evandro Chagas/FUNASA

Endereço para correspondência: Profa ${ }^{-1}$ Ana Yecê das Neves Pinto. Programa de Malária/Instituto Evandro Chagas/FUNASA. Av. Almirante Barroso 492, Marco, 66090-000 Belém, PA.

Tel: 5591 211-4439/211-4466/211-4432/214-2150, Fax: 5591 226-1284.

Recebido para publicação em 12/3/2002. 
sangüíneo, ingerir, além de outras formas sanguíneas parasitárias, os gametócitos de um doente com malária por $P$. vivax até 10 horas a partir do início do tratamento específico ${ }^{6}$, possibilitando a transmissão. Este fenômeno constitui um dos incentivos na busca de esquizonticidas sangüíneos mais eficazes, que atuem sobre todas as formas parasitárias e propiciem eficazmente a interrupção mais rápida na cadeia de transmissão. Pesquisas prévias mostram que os derivados da artemisinina constituem atualmente, os compostos mais eficazes no desaparecimento da sintomatologia e da parasitemia do portador de malária ${ }^{12458}$.

Esse estudo utilizou oito esquemas terapêuticos diferentes. Em dois dos grupos foi utilizada como esquizonticida sangüíneo a cloroquina em dose única de $10 \mathrm{mg}$ por $\mathrm{kg}$ de peso corporal e nos outros seis grupos, o artesunato por via oral em diferentes doses por apenas um dia. Associada a essas drogas foi utilizada a primaquina durante cinco ou sete dias, em todos os grupos.

\section{PACIENTES E MÉTODOS}

Pacientes atendidos no ambulatório do Programa de Malária do Instituto Evandro Chagas (Belém/PA). O estudo caracterizou-se por ser aberto, prospectivo e randomizado. Foram utilizados os seguintes critérios de inclusão: 1) hemoscopia positiva para $P$. vivax (gota espessa corada pelo método de Walker ${ }^{11}$ ); 2) idade a partir de 14 anos; 3) peso corporal acima de 40kg; 4) concordância em permanecer na Área Metropolitana de Belém por pelo menos seis meses a partir do início do tratamento; 5) consentimento para participação no estudo. Como critérios de exclusão foram considerados: 1) presença de complicações conseqüentes à malária ${ }^{10}$; 2) concomitância de outras enfermidades agudas e/ou crônico-degenerativas; 3) gestantes e/ou nutrizes.

Foram alocados 240 pacientes em lista randomizada e distribuídos em 8 esquemas terapêuticos, sendo 30 em cada grupo (Tabela 1).

Critérios de avaliação. A avaliação do quadro clínico, surgimento de possíveis efeitos colaterais, fornecimento da medicação específica e realização da

Tabela 1 - Grupos terapêuticos estudados, drogas, doses e tempo de tratamento.

\begin{tabular}{|c|c|c|}
\hline Grupos & Droga/dose no $1^{\circ}$ dia & $\begin{array}{c}\text { Uso da primaquina * } \\
\text { tempo (dias) }\end{array}$ \\
\hline A & artesunato 1 comprimido (50mg) $12 / 12$ horas & 7 \\
\hline B & artesunato 2 comprimidos (100mg) 12/12 horas & 7 \\
\hline C & artesunato 2 comprimidos $(100 \mathrm{mg})$ na hora zero $(\mathrm{H} 0)$ e 1 comprimido $(50 \mathrm{mg})$ na hora $12(\mathrm{H} 12)$ & 7 \\
\hline $\mathrm{D}$ & artesunato 1 comprimido $(50 \mathrm{mg})$ de $12 / 12$ horas & 5 \\
\hline$E$ & artesunato 2 comprimidos $(100 \mathrm{mg})$ de $12 / 12$ horas & 5 \\
\hline $\mathrm{F}$ & artesunato 2 comprimidos ( $100 \mathrm{mg})$ na $\mathrm{H} 0$ e 1 comprimido $(50 \mathrm{mg})$ na $\mathrm{H} 12$ & 5 \\
\hline G & aloroquina 4 comprimidos $(600 \mathrm{mg})$ em dose única & 7 \\
\hline $\mathrm{H}$ & aloroquina 4 comprimidos $(600 \mathrm{mg})$ em dose única & 5 \\
\hline
\end{tabular}

hemoscopia foram feitos diariamente. As drogas foram administradas sob supervisão da equipe. A partir de dois exames hemoscópicos negativos em dois dias consecutivos, a pesquisa de plasmódio era realizada apenas no oitavo dia após tratamento. A partir daí, os pacientes foram orientados a retornarem mensalmente ao ambulatório nos dias D30, D60, D90, D120, D150 e D180 ou excepcionalmente em qualquer dia, caso manifestassem novamente sintomatologia (febre).

Definições. Cura radical: pacientes acompanhados por pelo menos 180 dias, sem que tenham apresentado reaparecimento da parasitemia e/ou da sintomatologia malárica; não cura: pacientes tratados, apresentando involução da sintomatologia e lâminas negativas em dois dias consecutivos, que, porém, apresentaram durante o acompanhamento nova sintomatologia e hemoscopia positiva, estando incluídos aqui os casos de recaídas; controle incompleto: pacientes que terminaram o tratamento, porém não foram acompanhados por 180 dias; efeito colateral: qualquer sinal ou sintoma que tenha surgido ou exacerbado a partir do início do tratamento.

\section{RESULTADOS}

Foram alocados 240 pacientes em lista randomizada, sendo 30 pacientes em cada um dos oito grupos terapêuticos. Seis pacientes foram afastados do estudo em virtude da ocorrência de erro nas dosagens dos medicamentos antimaláricos. As principais características dos 234 pacientes, incluídos nos grupos terapêuticos estudados, podem ser observadas na Tabela 2.
Os grupos foram homogêneos quanto a parasitemia assexuada inicial, que variou de 10 a 40.000 formas assexuadas de $P$. vivax $/ \mathrm{mm}^{3}$ de sangue. A idade dos pacientes variou de 14 a 77 anos e em apenas $105(44,8 \%)$ pacientes foi possível a aferição do peso corporal (amplitude de 42 a $112 \mathrm{~kg}$, média $=62,5 \mathrm{~kg}$ ), não havendo diferenças significativas quanto a estas 
Tabela 2 - Principais características referentes aos 234 pacientes incluídos em oito grupos terapêuticos estudados.

\begin{tabular}{|c|c|c|c|c|c|c|c|c|}
\hline \multirow[b]{2}{*}{ Características } & \multicolumn{8}{|c|}{ Grupos terapêuticos } \\
\hline & A & B & C & D & $\mathrm{E}$ & $\mathrm{F}$ & G & $\mathrm{H}$ \\
\hline Pacientes $\left(\mathrm{n}^{\circ}\right)$ & 30 & 30 & 30 & 30 & 26 & 28 & 30 & 30 \\
\hline Média de idade (anos) & 31,8 & 33,0 & 36,9 & 29,7 & 32,3 & 33,0 & 36,6 & 29,1 \\
\hline Sexo masculino (\%) & 93,3 & 66,7 & 83,3 & 53,3 & 65,4 & 78,6 & 70,0 & 83,3 \\
\hline MGPA* $^{*}$ & 2,004 & 1,663 & 782 & 1,510 & 1,803 & 1,563 & 1,349 & 1,722 \\
\hline Cura radical & 22 & 19 & 19 & 21 & 15 & 15 & 24 & 22 \\
\hline Não cura & 0 & 1 & 5 & 4 & 5 & 5 & 1 & 4 \\
\hline Controle incompleto & 8 & 10 & 6 & 5 & 6 & 8 & 5 & 4 \\
\hline \multicolumn{9}{|l|}{ Pacientes com exame } \\
\hline negativo até 48h (\%) & $96,7^{\star *}$ & 100,0 & 100,0 & 100,0 & $96,2^{\star *}$ & 100,0 & 76,7 & 60,0 \\
\hline \multicolumn{9}{|l|}{ Pacientes assintomáticos } \\
\hline em 48h (\%) & 63,3 & 56,7 & 60,0 & 63,3 & 53,8 & 64,3 & 50,0 & 40,0 \\
\hline
\end{tabular}

variáveis entre os pacientes distribuídos nos grupos de estudo terapêuticos (Kruskal Wallis-H: respectivamente $p=0,3739$ e 0,4416).

Dos pacientes que completaram o controle de cura por 180 dias, a cura foi observada em $92,3 \%$ dos pacientes que tomaram primaquina em dose diária dobrada por sete dias (grupos $A+B+C+G$ ) e em $80,2 \%$ dos que fizeram uso desta droga em dose diária dobrada por cinco dias (grupos $D+E+F+H$ ) (quiquadrado: $p=0,0372$ ). $O$ controle de cura por 180 dias não pôde ser efetuado em $23,3 \%$ dos pacientes que tomaram primaquina por sete dias e em $19,3 \%$ dos que fizeram uso de primaquina por cinco dias.
Todos os 174 pacientes que tomaram artesunato via oral apresentaram negativação da parasitemia assexuada em até 48 horas (D2) a partir do início da terapêutica específica. Dos pacientes que fizeram uso de cloroquina apenas $68,3 \%$ apresentaram exame negativo durante o mesmo período (Teste Exato de Fisher: $p<0,01)$. A velocidade média da negativação da parasitemia nos pacientes tratados com artesunato via oral foi de 32,1 horas, e os que utilizaram cloroquina foi de 51,7 horas (Kruskal Wallis: $p<0,01$ ) (Figura 1).

Dos pacientes que foram tratados com artesunato, $60,3 \%$ ficaram assintomáticos da tríade malárica febre, calafrio e cefaléia até 48 horas a partir do início

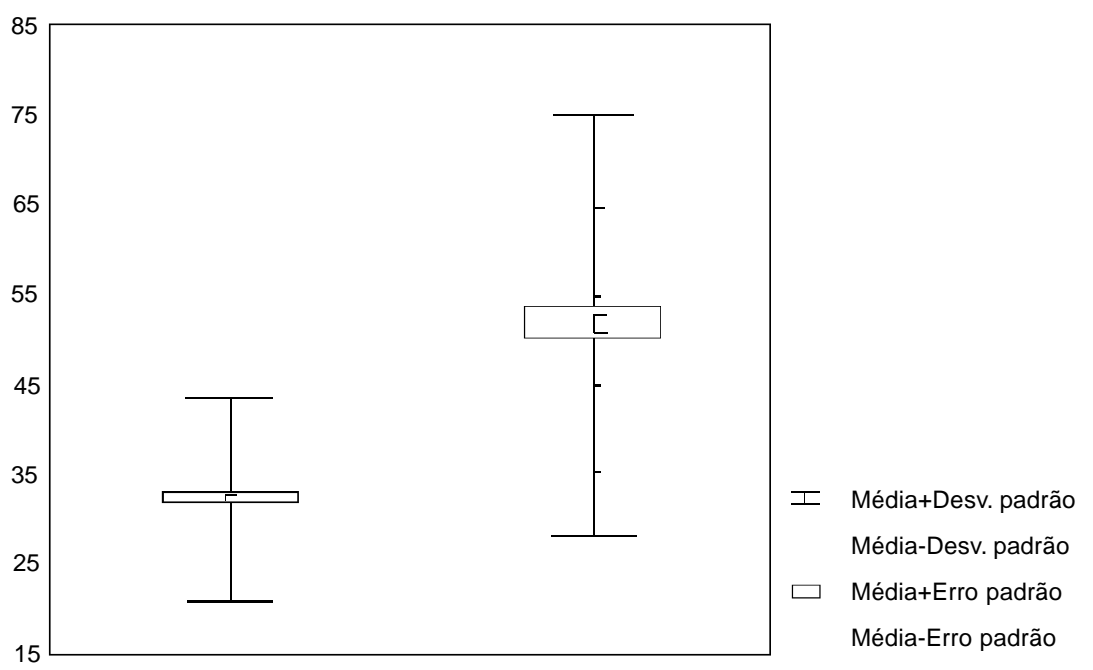

Figura 1 - Velocidade (em horas) da negativação parasitêmica dos pacientes com malária por $\mathrm{P}$. vivax submetidos ao tratamento com artesunato VO ou cloroquina,
Belém,

do tratamento, enquanto dos indivíduos que tomaram cloroquina apenas $45 \%$ ficaram assintomáticos durante o mesmo período. Os principais efeitos colaterais encontrados foram prurido (13/234), colúria
(8/234), epigastralgia (3/234) e diarréia (2/234), não havendo diferenças na apresentação dos efeitos colaterais nos diversos grupos. 


\section{DISCUSSÃO}

Os esquemas antimaláricos de mínima duração têm sido alvo constante de pesquisa como solução para a adesão mais efetiva dos doentes ao tratamento, principalmente quando se refere à Amazônia, onde o acesso ao tratamento é dificultado nas áreas malarígenas mais longínquas.

Os esquemas operacionais utilizados no presente trabalho mostraram eficácia, com percentuais de recidivas de $11,5 \%$, semelhantes aos resultados encontrados por Silva et $\mathrm{a}^{/ 14}$, usando primaquina em dose padrão $(0,25 \mathrm{mg} / \mathrm{kg}$ de peso corporal por dia) durante cinco dias ${ }^{14}$. Nos pacientes que fizeram uso do esquema $\mathrm{G}$ (cloroquina em dose única associado a primaquina por sete dias) observou-se $3,3 \%$ de respostas desfavoráveis, estando este percentual muito abaixo dos resultados encontrados em áreas não endêmicas usando o esquema clássico de cloroquina e primaquina em pacientes com malária por $P$. vivax ${ }^{3}$. Neste trabalho o uso do esquema clássico de cloroquina $25 \mathrm{mg} / \mathrm{kg}$ de peso corporal divididos em três dias, associado a primaquina na dose de $0,25 \mathrm{mg} / \mathrm{kg}$ de peso por quatorze dias, mostrou índices de $24 \%$ de recaídas, enquanto Silva et a/ ${ }^{10}$ no período de 1981 e 1982 na Amazônia oriental (Maranhão) relataram 5,6\% de recaídas usando o esquema clássico ${ }^{14}$. Assim, os autores sugerem que a utilização de esquemas terapêuticos mais operacionais não eleva o risco de obtenção de respostas ineficazes.

Sharma et a ${ }^{\prime 3}$ trataram 1.520 pacientes com malária por $P$. vivax na Índia usando dose única de $600 \mathrm{mg}$ de cloroquina associada a primaquina ( $15 \mathrm{mg} / \mathrm{dia})$, ou seja, cloroquina nas mesmas doses utilizadas nos esquemas $\mathrm{G}$ e $\mathrm{H}$, porém primaquina em dose simples por cinco dias observaram que as recaídas durante o primeiro ano de acompanhamento totalizaram 2,6\% ${ }^{9}$. Em outro estudo realizado na Índia, foram tratadas 1.345 pessoas com malária por $P$. vivax usando cloroquina em dose total de $900 \mathrm{mg}$ dividida em dois dias associada à primaquina $15 \mathrm{mg} /$ dia durante cinco dias, sendo encontradas recaídas em $6,9 \%$ dos casos $^{15}$. Estes resultados demonstram que as recaídas ocorrem em diferentes percentuais conforme as diferentes áreas malarígenas. Considerando estas diferenças regionais de aparecimento de recaídas e as diferentes respostas dos plasmódios aos antimaláricos, os autores sugerem que os dados ora apresentados, se prestam para aplicação limitada ao Estado do Pará, área de procedência dos casos estudados.

Os pacientes tratados com a primaquina por sete dias obtiveram maior percentual de cura quando comparados aos que usaram esta droga por cinco dias, independente da droga esquizonticida empregada.

O artesunato demostrou ser droga mais veloz que a cloroquina tanto na negativação da parasitemia assexuada, quanto na cura clínica, indicando ser uma excelente opção em casos especiais de impossibilidade do uso de cloroquina, corroborando os resultados de outros estudos feitos com esta droga no tratamento de malária vivax ${ }^{2712}$.

O esquema G deverá ser usado em outros estudos, que tenham como finalidade compará-lo ao esquema clássico, para que se possa avaliar o percentual de eficácia entre os dois tratamentos empregados.

Os autores corroboram as atuais indicações dos novos esquemas terapêuticos encurtados em malária contidos no Manual de Terapêutica de Malária lançado em 2001.

\section{AGRADECIMENTOS}

Ao Dr. Manuel Ayres pela colaboração na análise estatística dos dados. A todos os técnicos do Programa de Malária do Instituto Evandro Chagas e a todos os voluntários que participaram deste trabalho.

\section{REFERÊNCIAS BIBLIOGRÁFICAS}

1. Alecrim MG, Carvalho LM, Fernandes MC, Andrade SD, Loureiro AC, Arcanjo AR, Alecrim WD. Revista da Sociedade Brasileira de Medicina Tropical 33:163-168, 2000.

2. Batty KT, Le AT, llett KF, Nguyen PT, Powell SM, Nguyen $\mathrm{CH}$, Truong XM, Vuong VC, Huynh VT, Tran QB, Nguyen VM, Davis TM. A pharmacokinetic and pharmacodynamic study of artesunate for vivax malaria. American Journal of Tropical Medicine and Hygiene 59:823-827, 1998.

3. Boulos $M$, Amato Neto V, Dutra AP. Análise da freqüência de recaídas de malária por Plasmodium vivax em região não endêmica (São Paulo, Brasil). Revista do Instituto de Medicina Tropical de São Paulo 33:143-146, 1991.

4. Elhassan IM, Satti GH, Ali AE, Fadul I, Elkhalifa AA, Abedelrahim AM, Ming C, Theander TG. The efficacy of artemether in the treatment of Plasmodium falciparum malaria in Sudan. Transactions of the Royal Society of Tropical Medicine and Hygiene 87:685-686, 1993.
5. Fundação Nacional de Saúde. Manual de terapêutica da malária/ Colaboração de Agostinho Cruz Marques [et al]. Ministério da Saúde, Brasília, DF, 2001.

6. Klein TA, Tada MS, Lima JB, Tang AT. Infection of Anopheles darlingi fed on patients infected with Plasmodium vivax before and during treatment with chloroquine plus primaquine in Costa Marques, Rondônia, Brazil. Memórias do Instituto Oswaldo Cruz 87:191-195, 1992.

7. Li GQ, Guo XB, Fu LC, Jian HX, Wang XH. Clinical trial of artemisin and its derivates in the treatment of malaria in China. Transactions of Royal Society in Tropical Medicine and Hygiene 88 (supl I): 55-56, 1994.

8. Looareesuwan $S$, Viravan $C$, Vanijanonta $S$, Wilairatana $P$, Suntharasamai P, Charoenlarp P, Arnold K, Kyle D, Canfield C, Webster K. Randomized trial of artesunate and mefloquine alone and in sequence for acute uncomplicated falciparum malaria. Lancet 339:821-824, 1992. 
9. Marques AC, Cárdenas GH. Combate à malária no Brasil situação atual e perspectivas. Revista da Sociedade Brasileira de Medicina Tropical 27:91-108, 1994.

10. Organização Mundial da Saúde. Tratamento da Malária Grave e Complicada-Condutas Práticas. Genebra, 1991.

10. Organizacion Panamericana de la Salud. Manual de técnicas básicas para um laboratório de salud. Publicacion Científica $\mathrm{n}^{0}$ 439, Washington, 1983.

11. Pukrittayakamee S, Chantra A, Simpson JÁ, Vanijanonta S, Clemens R, Looareesuwan $S$, White NJ. Therapeutic response to different antimalarial drugs in vivax malaria. Antimicrobial Agents Chemotherapeutic. 44:1680-1685, 2000.
12. Sharma RC, Gautam AS, Orlov V, Sharma VP. Relapse pattern of Plasmodium vivax in Kheda District, Gujarat. Indian Journal of Malariology 27:95-99, 1990.

13. Silva AR, Silva CMP, Carvalho Branco MRF, Branco Filho JRC. Resultado do uso de um esquema terapêutico para Plasmodium vivax em 5 dias em 3 municípios da llha de São Luis, estado do Maranhão, Brasil. Revista da Sociedade Brasileira de Medicina Tropical 22:131-136, 1989.

14. Sinha S, Dua VK, Sharma VP. Efficacy of 5 day radical treatment of primaquine in Plasmodium vivax cases at the BHEL Industrial Complex, Hardwar (U.P). Indian Journal of Malariology 26:8386, 1989. 